INPLASY

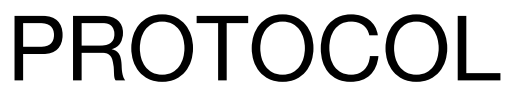

To cite: Ran et al. Epidural steroids following

Percutaneous Endoscopic Interlaminar Discectomy : A protocol for systematic review and meta-analysis. Inplasy protocol 2020100085. doi: 10.37766/inplasy2020.10.0085

Received: 22 October 2020

Published: 22 October 2020

Corresponding author: Qiang Ran

403158567@qq.com

Author Affiliation:

Chengdu University of

Traditional Chinese Medicine

Support: National Key

Research.

Review Stage at time of this submission: Preliminary searches.

Conflicts of interest:

The authors report no conflicts of interest.

\section{Epidural steroids following Percutaneous Endoscopic Interlaminar Discectomy : A protocol for systematic review and meta-analysis}

\author{
Ran, Q1; Yu, Y2; Li, T3; Fan, X².
}

Review question / Objective: P:Percutaneous Endoscopic Interlaminar Discectomy I:Epidural steroids C: saline injection O:relief of pain S:RCT.

Condition being studied: Percutaneous Endoscopic Interlaminar Discectomy(PEID) has been widely used in the treatment of Lumbar disc herniation and spinal stenosis, epidural steroids following PEID in an effort to reduce the incidence and duration of postoperative pain. Although steroids are widely thought to reduce the effect of surgical trauma,the observation index is not uniform, so the issue is still controversial. Therefore, the purpose of this paper is to systematically evaluate the efficacy and safety of local epidural steroids application following PEID.

INPLASY registration number: This protocol was registered with the International Platform of Registered Systematic Review and Meta-Analysis Protocols (INPLASY) on 22 October 2020 and was last updated on 22 October 2020 (registration number INPLASY2020100085).

\section{INTRODUCTION}

Review question / Objective: P:Percutaneous Endoscopic Interlaminar Discectomy I:Epidural steroids C: saline injection O:relief of pain S:RCT.
Condition being studied: Percutaneous Endoscopic Interlaminar Discectomy(PEID) has been widely used in the treatment of Lumbar disc herniation and spinal stenosis, epidural steroids following PEID in an effort to reduce the incidence and duration of postoperative pain. Although steroids are 
widely thought to reduce the effect of surgical trauma,the observation index is not uniform, so the issue is still controversial. Therefore, the purpose of this paper is to systematically evaluate the efficacy and safety of local epidural steroids application following PEID.

\section{METHODS}

Participant or population: Randomized controlled trials (RCTs) that evaluated the efficacy and safety of epidural steroids for the treatment PEID.

Intervention: Epidural steroids following Percutaneous Endoscopic Interlaminar Discectomy.

Comparator: Saline injection following Percutaneous Endoscopic Interlaminar Discectomy.

Study designs to be included: RCT.

Eligibility criteria: Patients in chosen trials had been epidural steroids Injected following PEID, providing appropriate management with outcome evaluations of 3 months or longer and statistical evaluations will be reviewed. Reports without appropriate diagnosis, nonsystematic reviews, book chapters, and case reports will be excluded.

Information sources: PubMed, Embase, Medline, Chinese National Knowledge Infrastructure, Chinese Biomedical Literature Database, Web of Science, Wan Fang Database, Cochrane Library.Patients in chosen trials had been epidural steroids Injected following PEID, providing appropriate management with outcome evaluations of 3 months or longer and statistical evaluations will be reviewed. Reports without appropriate diagnosis, non-systematic reviews, book chapters, and case reports will be excluded.

Main outcome(s): The primary outcome parameter is relief of pain. The secondary outcome measure is functional status improvement. Postoperative complications will be observed in the meantime.
Quality assessment / Risk of bias analysis: The literature of randomized controlled trials was evaluated with Cochrane bias risk assessment form, which included: 1) the generation of random sequences; 2) allocation concealment; 3) blind method application; 4) blind method evaluation; 5) Data Integrity; 6) selective reporting of findings; 7) other biases.

Strategy of data synthesis: The literatures will be reviewed with the Cochrane (Cochrane Manual V.5.1.0)Bias Risk Assessment. The funnel chart will be used to assess the reporting bias. When the number of studies is sufficient, we will use the Eger method to test. The unit of analysis will be conducted by the independent reviewers. The missing data will be complemented by independent reviewers through contacting with the corresponding author. The funnel charts will be used to assess reporting biases.

Subgroup analysis: Subgroup analysis We will observe the source of considerable heterogeneity by subgroup analysis based on variations in study and patient characteristics, study quality, different interventions, comparators, and outcomes.

Sensibility analysis: If necessary, the sensitivity analysis will be used to assess the effect of each study on the random effects model. Studies with a high risk of bias will be excluded to permit the evaluation of the robustness and reliability of the analysis.

\section{Country(ies) involved: China.}

Keywords: epidural,steroids, percutaneous endoscopic interlaminar discectomy, protocol, systematic review.

Contributions of each author:

Author 1 - Qiang Ran.

Author 2 - Yang Yu.

Author 3 - Tong Li.

Author 4 - Xiaohong Fan. 